\title{
Review
}

Lisa J. Hayden, Sarah Y. Jeong* and Carol A. Norton

\section{An Analysis of Factors Affecting Mature Age Students' Academic Success in Undergraduate Nursing Programs: A Critical Literature Review}

DOI 10.1515/ijnes-2015-0086

Received November 12, 2015; revised September 24, 2016; accepted October 10, 2016

\begin{abstract}
The population of mature age students entering university nursing programs has steadily increased in both Australia and worldwide. The objective of the literature review was to explore how mature age students perform academically and to analyse the factors associated with their academic performance in nursing programs. A literature search was conducted in the following databases: CINAHL, ProQuest, Medline, Cochrane, Mosby's Index, Joanna Briggs Institute (JBI), and Scopus. Twenty-six (26) research papers published between 2000 and 2014 have met the selection criteria of this review. The key themes identified include; 1) ambiguity in definition of mature age and academic success, 2) age and academic success, 3) intrinsic factors (life experiences, emotional intelligence, and motivation and volition), and 4) extrinsic factors (peer, academic and family support; and learning style, components of the modules and mode of delivery). Current literature provides evidence that mature age nursing students perform at a higher level within the methodological issues discussed in this paper. Future research is warranted to advance the understanding of the complex relationship between extrinsic and intrinsic factors of mature age students and their academic success in higher education. Nursing educators will benefit from novel evidence, ideas and opportunities to explore and implement in nursing education.
\end{abstract}

\footnotetext{
*Corresponding author: Sarah Y. Jeong, School of Nursing \& Midwifery, The University of Newcastle, PO Box 127, Ourimbah, NSW 2258, Australia, E-mail: Sarah.Jeong@newcastle.edu.au Lisa J. Hayden, University of Newcastle, Teaching and Research Unit, Gosford Hospital, and Central Coast Local Health District, Gosford, NSW 2250, Australia, E-mail: Lisa.Hayden@newcastle.edu.au Carol A. Norton, School of Nursing \& Midwifery, The University of Newcastle, PO Box 127, Ourimbah, NSW 2258, Australia,

E-mail: Carol.Norton@newcastle.edu.au
}

Keywords: mature age students, adult learners, academic success, nursing education

\section{Introduction}

A large portion of mature age students comprises the student population on university campuses globally (OECD, 2012). Although what constitutes a mature age student is ambiguous, in Australia, $41 \%$ of university students are aged 25-64 years old and 59\% are aged between 15 and 24 years old (Australian Bureau of Statistics (ABS), 2013). There has been a steady increase in the number of mature age students in nursing programs (Kenny et al., 2007). Mature age students are drawn to nursing because of the human-altruistic values and the financial security offered in the profession (Watson, 2008). Gaining admission to a nursing program at a university is one thing, achieving academic success in a program of study is another, given the additional responsibilities one has as a mature age student (Sogunro, 2015). There have been numerous attempts to explain how to motivate students and sustain their ability to succeed at the university level (Bannier, 2010; Malacinski \& Winterman, 2012; Wilde, 2012). However, the issues in relation to mature age nursing students' performance and success in Bachelor programs are complicated and require attention from university educators.

\section{Background}

Nursing is one of the most popular university degrees chosen by mature age students (Australian Bureau of Statistics (ABS), 2013). Various studies over the past two decades have demonstrated a direct correlation between mature age students, academic achievement and the benefits of their academic success. Kenny and her colleagues (2011) found lower rates of attrition and higher levels of 
academic performance, compared to younger students and suggested that mature age students should be targeted for entry into nursing education due to their high success rate. Conversely, the successful completion (graduation) rate of students who are aged 21 years and over is lower at $78.1 \%$ compared to $87.5 \%$ for students who are less than 21 years old (University of Newcastle, 2014). It is also important to expect that mature age students may have shorter career spans and decreased working hours per week, ultimately intensifying the nursing shortage (Hickey, Sumsion, \& Harrison, 2010).

In an attempt to investigate this contradictory argument, various explanations were identified in the authors' preliminary literature review. For example, family responsibilities, stress, relationships and economic instability are the non-academic components that affect academic performance (Freitas \& Leonard, 2011). In another study, Ofori and Charleton (2002) found that selfefficacy and internal locus of control of mature age nursing students and their supportive environments are essential to their academic success. However, there is absence of detailed analysis of the characteristics of mature age nursing students and their supportive environments. There are inconsistent and contradictory assumptions and claims which require further investigation on the mature age students in nursing programs and their academic success.

The aims of the literature review were; (1) to explore how mature age students perform academically in undergraduate nursing programs; (2) to investigate the factors that affect mature age students' academic success, and (3) to discuss critically their relevance in undergraduate nursing education.

\section{Methodology}

The eligibility criteria for articles to be included in this review were that participants must include mature age nursing students, the research had to address an account of academic success, and the setting was to be only at a university level. The articles also had to be research papers only. Articles excluded were those published prior to 2000, non-English papers, non-research papers (i. e. discussions, literature reviews, and theoretical frameworks), papers focusing on the post graduate level, online programs, perceptions of other stakeholders (e.g. clinicians) and research settings other than university.

The strategy for this literature review consisted of a database search of peer-reviewed scholarly articles from
2000 to 2014. Seven (7) databases were searched: Cumulative Index to Nursing and Allied Health (CINHAL), ProQuest, Medline, Cochrane, Mosby's Index, Joanna Briggs Institute (JBI), and Scopus. The scope of the search was conducted from January 2000 and April 2014 for peer-reviewed, English written scholarly journals. The search included the keywords of mature age students, adult learners, academic success and nursing education and resulted in four hundred and seventyfour (474) journal articles. Due to the large number of articles, the articles were further narrowed by major subject categories: academic performance, student retention, student experiences, student dropouts, age factors, academic achievement, education-nursing, baccalaureate, education (general), nursing, and student attitudes. Duplicate articles and the journal articles that did not address the aim of literature review were excluded from the search. In addition, twelve (12) journal articles were obtained from a secondary informal document search.

In total, there were fifty-eight (58) articles that were assessed using Critical Review Forms and 'Guidelines for critical review - Quantitative studies' (Law et al., 1998a, 1998b) or 'Guidelines for critical review - Qualitative studies' (Letts et al., 2007a, 2007b). A total of twenty-six (26) of the fifty-eight (58) articles that described, examined, and evaluated the various reasons or factors that allow adult learners to succeed in university nursing programs were selected. There were thirteen (13) quantitative studies, seven (7) qualitative studies and six (6) mixed method studies included in the review. Figure 1 provides a decision flow diagram.

\section{Results}

The articles were analysed using thematic analysis by the primary and other authors. Discussion among the authors included an agreement of the themes and interpretation of the findings in the journal articles. This process of peer review ensured rigour and trustworthiness of thematic analysis.

Table 1 provides a summary of the literature reviewed. The key themes identified in this review include; 1) ambiguity in definition of mature age students and academic success, 2) age and academic success, 3) intrinsic factors (life experiences; and motivation and volition), and 4) extrinsic factors (peer, academic and family support; and learning style, course content and mode of delivery). 
Articles $(n=474)$ identified through database search: Literature Search Databases: CINHAL, ProQuest, Medline, Cochrane, Mosby's Index, Johanna Briggs Institute, and Scopus Search term keywords: mature age students, adult learners, academic success and nursing baccalaureate (Boolean/Phrase mode).

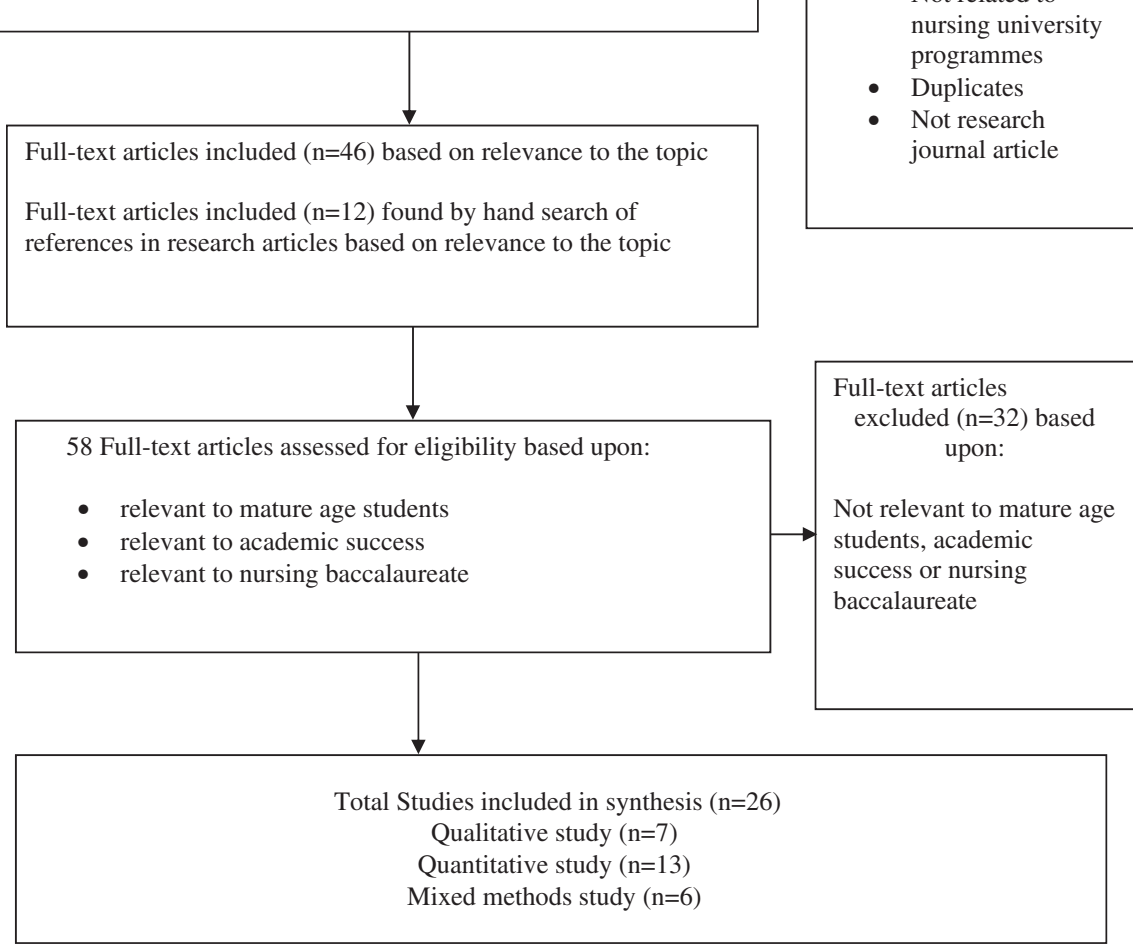

Figure 1: A decision flow diagram: mature age nursing students and academic success.

\section{Ambiguity in definition of 'mature age' and 'academic success'}

A review of the literature indicates that 12 of the 26 selected articles did not clearly define the term 'mature age' student (See Table 1). While two articles (Fleming, McKee, \& Huntley-Moore, 2011; O’Brien, Keogh, \& Neenan, 2009) defined mature students as individuals who are 23 years of age or over, five of the articles defined 'mature age' as over the age of 21 years (Boelen \& Kenny, 2009; Clarke, Miers, Pollard, \& Thomas, 2007; Drury, Francis, \& Chapman, 2008; Kenny et al., 2011; Trotter \& Cove, 2005). One article defined the range between 21 and 50 years old as mature age (Lee, Cholowksi, \& Williams, 2002). In the United Kingdom, mature age is defined as 26 years and older before the first year of university (Kevern \& Webb, 2004; McCarey, Barr, \& Rattray, 2007).

One article divided mature age students into two separate categories where 'mature age' students are
Articles $(n=428)$ excluded based upon:

- Not relevant to review aim of research

- Not related to nursing field

Not related to nursing university programmes journal article

Full-text article $(n=32)$ based

o mature age 
Table 1: A summary of literature reviewed.

\begin{tabular}{|c|c|c|c|c|c|}
\hline Author/Year/Country & $\begin{array}{l}\text { Definition: } \\
\text { mature age/ } \\
\text { academic } \\
\text { success }\end{array}$ & Aim & $\begin{array}{l}\text { Research design/ } \\
\text { sample/data } \\
\text { collection }\end{array}$ & Key findings & Limitations \\
\hline $\begin{array}{l}\text { 1. Anderson, E., \& } \\
\text { Thorpe, L. } \\
\text { /2008/United } \\
\text { Kingdom }\end{array}$ & No/No & $\begin{array}{l}\text { To evaluate } \\
\text { Interprofessional } \\
\text { Learning Event (IPE) } \\
\text { with different student } \\
\text { age groups in three } \\
\text { different universities. }\end{array}$ & $\begin{array}{l}\text { Mixed Method: } \\
\text { Quantitative }(n=754) \\
\text { health and social } \\
\text { students: Qualitative } \\
\text { ( } n=81) \text { : nursing } \\
\text { students }\end{array}$ & $\begin{array}{l}\text { Mature entrants } \\
\text { needed a more } \\
\text { demanding, } \\
\text { challenging \& relevant } \\
\text { set of learning } \\
\text { resources that are } \\
\text { sensitive to prior life } \\
\text { experience. }\end{array}$ & $\begin{array}{l}\text { No details of the } \\
\text { mature students } \\
\text { previous } \\
\text { engagement with } \\
\text { and experience of } \\
\text { interprofessional } \\
\text { values. }\end{array}$ \\
\hline $\begin{array}{l}\text { 2. Boelen, M., \& Kenny, } \\
\text { A./2009/ Australia }\end{array}$ & $\begin{array}{l}\text { Mature aged (>21 } \\
\text { years)/ No }\end{array}$ & $\begin{array}{l}\text { To evaluate one-week } \\
\text { transition science } \\
\text { course for enrolled } \\
\text { nurses to study at } \\
\text { university level course }\end{array}$ & $\begin{array}{l}\text { Quantitative: } \mathrm{n}=70 \\
\text { students (enrolled } \\
\text { nurses registered for } \\
\text { BN program) }\end{array}$ & $\begin{array}{l}\text { Students built social } \\
\text { networks, developed } \\
\text { learning strategies } \\
\text { and connected with } \\
\text { other students. }\end{array}$ & $\begin{array}{l}\text { Small sample size at } \\
\text { one university within } \\
\text { a short period of } \\
\text { time. }\end{array}$ \\
\hline $\begin{array}{l}\text { 3. Bowden, J. } \\
\text { /2008/United } \\
\text { Kingdom. }\end{array}$ & No/No & $\begin{array}{l}\text { To explore factors } \\
\text { affecting the students } \\
\text { to leave the } \\
\text { programme }\end{array}$ & $\begin{array}{l}\text { Mixed Method: } \\
\text { Quantitative: Survey } \\
(n=46) \text { Qualitative: } \\
\text { interviews }(n=8) \text { Age } \\
\text { range: } 19-42\end{array}$ & $\begin{array}{l}\text { Mature students } \\
\text { found tutors to be } \\
\text { most helpful followed } \\
\text { by Peers, family and } \\
\text { friends. }\end{array}$ & $\begin{array}{l}\text { Small sample size, } \\
\text { eight interviews-not } \\
\text { a good } \\
\text { representative of the } \\
\text { cohort }\end{array}$ \\
\hline $\begin{array}{l}\text { 4. Clarke, B., Miers, M., } \\
\text { Pollard, K., \& } \\
\text { Thomas, J. /2007/ } \\
\text { United Kingdom }\end{array}$ & $\begin{array}{l}\text { Mature aged (>21 } \\
\text { years)/No }\end{array}$ & $\begin{array}{l}\text { To examine group } \\
\text { dynamics between } \\
\text { interprofessionals } \\
\text { undergraduate groups } \\
\text { in health and social } \\
\text { care. }\end{array}$ & $\begin{array}{l}\text { Qualitative: Non- } \\
\text { Participant } \\
\text { Observations/ Focus } \\
\text { Groups/ Semi- } \\
\text { structured Student } \\
\text { Interviews } \mathrm{n}=33 \text {, Age } \\
\text { Range: } 18-40+\end{array}$ & $\begin{array}{l}\text { Mature age students } \\
\text { play a more active } \\
\text { role; take more } \\
\text { responsibility for } \\
\text { discussion, and aware } \\
\text { of their ability to learn } \\
\text { through discussion. }\end{array}$ & $\begin{array}{l}\text { Age differences } \\
\text { created difficulties } \\
\text { for group: reason } \\
\text { not discussed }\end{array}$ \\
\hline $\begin{array}{l}\text { 5. Drury, V., Francis, K., } \\
\text { \& Chapman, Y. } \\
\text { /2008/ Australia }\end{array}$ & $\begin{array}{l}\text { Mature aged (>21 } \\
\text { years)/No }\end{array}$ & $\begin{array}{l}\text { To understand how } \\
\text { mature aged people } \\
\text { reconstruct } \\
\text { themselves as nursing } \\
\text { students }\end{array}$ & $\begin{array}{l}\text { Qualitative: Grounded } \\
\text { theory } n=14 \text { mature } \\
\text { age nursing students }\end{array}$ & $\begin{array}{l}\text { Mature Age students } \\
\text { have different needs } \\
\text { such as academic and } \\
\text { pastoral support. }\end{array}$ & $\begin{array}{l}\text { Qualitative } \\
\text { constructed } \\
\text { grounded theory: } \\
\text { findings cannot be } \\
\text { generalised. }\end{array}$ \\
\hline $\begin{array}{l}\text { 6. El Ansari, W. } \\
\text { /2002/United } \\
\text { Kingdom }\end{array}$ & $\begin{array}{l}\text { Mature students } \\
21 \pm 25 ; \& \text { older } \\
\text { mature students } \\
>25 / \text { No }\end{array}$ & $\begin{array}{l}\text { To investigate the } \\
\text { effects of } \\
\text { demographic } \\
\text { variables on students' } \\
\text { perceptions and } \\
\text { satisfaction }\end{array}$ & $\begin{array}{l}\text { Quantitative: Cross- } \\
\text { Sectional Survey } \\
\mathrm{n}=460 \text { student } \\
\text { participants Mean } \\
\text { student age: } 30 \text { years } \\
\text { (range } 18 \pm 53 \text { years) }\end{array}$ & $\begin{array}{l}\text { Age was both } \\
\text { positively and } \\
\text { significantly } \\
\text { correlated with the } \\
\text { grade achieved } \\
(r=0.277, \\
p<0.0001) .\end{array}$ & $\begin{array}{l}\text { Limitations inherent } \\
\text { in questionnaire } \\
\text { Design; Self- } \\
\text { selection and self- } \\
\text { report }\end{array}$ \\
\hline $\begin{array}{l}\text { 7. Fernandez, R. et al. } \\
\text { /2012/Australia }\end{array}$ & $\begin{array}{l}\text { No/GPA obtained } \\
\text { at the end of six } \\
\text { months }\end{array}$ & $\begin{array}{l}\text { To examine } \\
\text { association between } \\
\text { emotional intelligence } \\
\text { and learning } \\
\text { strategies on } \\
\text { academic } \\
\text { performance }\end{array}$ & $\begin{array}{l}\text { Quantitative: } \\
\text { Prospective Survey } \\
\text { Design } n=81 \text { 1st year } \\
\text { nursing students } \\
\text { Mean age: } 29 \text { years } \\
\text { old. }\end{array}$ & $\begin{array}{l}\text { El is a significant } \\
\text { predictor of academic } \\
\text { achievement (b } \\
=0.25 ; p=0 \text { AE023). }\end{array}$ & $\begin{array}{l}\text { All women and } \\
\text { international } \\
\text { students Self-report }\end{array}$ \\
\hline $\begin{array}{l}\text { 8. Fleming, S., McKee, } \\
\text { G. /2005/Ireland }\end{array}$ & $\begin{array}{l}>23 \text { years of age } \\
\text { or over/No }\end{array}$ & $\begin{array}{l}\text { To develop and } \\
\text { evaluate 'Welcome } \\
\text { Programme' for } \\
\text { mature students }\end{array}$ & $\begin{array}{l}\text { Quantitative: } \\
\text { Questionnaires } \\
\text { Average age: } 31.7 \\
\text { years old }\end{array}$ & $\begin{array}{l}\text { Mature age students } \\
\text { identified need for } \\
\text { knowledge of course, } \\
\text { biology and financial } \\
\text { issues. }\end{array}$ & \\
\hline
\end{tabular}


Table 1: (continued)

\begin{tabular}{|c|c|c|c|c|c|}
\hline Author/Year/Country & $\begin{array}{l}\text { Definition: } \\
\text { mature age/ } \\
\text { academic } \\
\text { success }\end{array}$ & Aim & $\begin{array}{l}\text { Research design/ } \\
\text { sample/data } \\
\text { collection }\end{array}$ & Key findings & Limitations \\
\hline $\begin{array}{l}\text { 9. Freitas \& Leonard/ } \\
\text { 2011/United States }\end{array}$ & No/No & $\begin{array}{l}\text { To identify the factors } \\
\text { contributing to } \\
\text { student nurse success }\end{array}$ & $\begin{array}{l}\text { Quantitative: Survey } \\
\mathrm{n}=190 \text { entry-level } \\
\text { students Average age: } \\
28.7 \text { years. }\end{array}$ & $\begin{array}{l}\text { Older respondents } \\
\text { reported greater } \\
\text { ability to meet their } \\
\text { needs than did } \\
\text { younger respondents. }\end{array}$ & $\begin{array}{l}\text { Small sample size } \\
\text { No significant } \\
\text { relationship } \\
\text { between GPA and } \\
\text { any of the } \\
\text { demographic data. }\end{array}$ \\
\hline $\begin{array}{l}\text { 10. Jones-Schenk, J. \& } \\
\text { Harper, M.G./ } \\
\text { 2014/United States }\end{array}$ & No/GPA & $\begin{array}{l}\text { To determine the } \\
\text { relationship between } \\
\text { students' El and their } \\
\text { likelihood of success } \\
\text { in nursing school }\end{array}$ & $\begin{array}{l}\text { Quantitative: Survey } \\
\mathrm{n}=116 \text { students } \\
\text { Mean age: } 36.21\end{array}$ & $\begin{array}{l}\text { El characteristics of } \\
\text { successful students } \\
\text { scored significantly } \\
\text { higher on the } \\
\text { Intrapersonal scale } \\
\text { and the Stress } \\
\text { Tolerance subscale. }\end{array}$ & $\begin{array}{l}\text { Study Participants } \\
\text { are enrolled in an } \\
\text { online program. }\end{array}$ \\
\hline $\begin{array}{l}\text { 11. Kenny, A. et al. } \\
\text { /2011/ Australia }\end{array}$ & $\begin{array}{l}\text { Defined as }>21 \\
\text { years of age/No }\end{array}$ & $\begin{array}{l}\text { To identify specific } \\
\text { needs of mature age } \\
\text { nursing students }\end{array}$ & $\begin{array}{l}\text { Mixed Method: Action } \\
\text { Research Study }\end{array}$ & $\begin{array}{l}\text { Mature age students } \\
\text { need specific } \\
\text { information directed } \\
\text { towards their } \\
\text { learning. }\end{array}$ & $\begin{array}{l}\text { Questionnaire not } \\
\text { tested for reliability } \\
\text { and validity }\end{array}$ \\
\hline $\begin{array}{l}\text { 12. Kevern, J. \& C. } \\
\text { Webb/2004/ United } \\
\text { Kingdom }\end{array}$ & $\begin{array}{l}>26 \text { years before } \\
\text { the first year } \\
\text { course/No }\end{array}$ & $\begin{array}{l}\text { To explore the } \\
\text { experiences of mature } \\
\text { nursing students in } \\
\text { higher education }\end{array}$ & $\begin{array}{l}\text { Qualitative: } \\
\text { Exploratory Research } \\
\mathrm{n}=32 \text { female } \\
\text { participants Mean } \\
\text { age: } 41 \text { years old }\end{array}$ & $\begin{array}{l}\text { Maturity and life } \\
\text { experience provides } \\
\text { useful time- } \\
\text { management and } \\
\text { multitasking skills }\end{array}$ & $\begin{array}{l}\text { Gender specific No } \\
\text { generalizable due to } \\
\text { small sample size \& } \\
\text { qualitative }\end{array}$ \\
\hline $\begin{array}{l}\text { 13. Lee, W., et al. } \\
\text { /2002/ Australia }\end{array}$ & $\begin{array}{l}\text { Between } 21 \text { and } \\
50 \text { years old / No }\end{array}$ & $\begin{array}{l}\text { To explore the } \\
\text { perceived } \\
\text { characteristics of } \\
\text { effective clinical } \\
\text { educators as rated by } \\
\text { students and } \\
\text { educators }\end{array}$ & $\begin{array}{l}\text { Qualitative: Survey } \\
\text { 2nd year nursing } \\
\text { students }(n=104) \\
\text { Clinical educator } \\
(n=17) \text { Man age: } 24 \\
\text { years }\end{array}$ & $\begin{array}{l}\text { Mature age group } \\
\text { valued academic } \\
\text { guidance; need for } \\
\text { performance feedback }\end{array}$ & $\begin{array}{l}\text { Second year } \\
\text { students only Small } \\
\text { sample size }\end{array}$ \\
\hline $\begin{array}{l}\text { 14. Madigan, V. } \\
\text { /2006/Australia }\end{array}$ & $\begin{array}{l}\text { Mature-entry } \\
\text { students ( } \geq 20 \\
\text { years)/ GPA }\end{array}$ & $\begin{array}{l}\text { To identify factors } \\
\text { relating to academic } \\
\text { performance in } \\
\text { predicting first-year } \\
\text { academic success }\end{array}$ & $\begin{array}{l}\text { Quantitative: } \\
\text { Retrospective review: } \\
\text { Six predictors of } \\
\text { academic } \\
\text { performance were } \\
\text { examined } n=135\end{array}$ & $\begin{array}{l}\text { Mature aged students } \\
\text { pass and complete } \\
\text { the first year; } \\
\text { however, perform } \\
\text { better if they have } \\
\text { previous healthcare } \\
\text { experience }\end{array}$ & $\begin{array}{l}\text { Incomplete data re: } \\
\text { GPA for some } \\
\text { students. Small } \\
\text { sample size }\end{array}$ \\
\hline $\begin{array}{l}\text { 15. McCarey, M., et al. } \\
\text { /2007/ United } \\
\text { Kingdom }\end{array}$ & $\begin{array}{l}\geq 26 \text { years old } / \\
\text { year three } \\
\text { assignments and } \\
\text { examinations }\end{array}$ & $\begin{array}{l}\text { To explore if academic } \\
\text { performance in year } \\
\text { three be predicted } \\
\text { from: (a) entry } \\
\text { qualifications (b) } \\
\text { gender (c) age (d) } \\
\text { attendance (e) } \\
\text { academic } \\
\text { performance }\end{array}$ & $\begin{array}{l}\text { Quantitative: Student } \\
\text { record data Nursing } \\
\text { students }(n=154)\end{array}$ & $\begin{array}{l}\text { Mature age students } \\
\text { achieved better marks } \\
\text { in course work and } \\
\text { examinations; more } \\
\text { motivated to succeed; } \\
\text { committed to study; } \\
\text { and more willing to } \\
\text { participate in } \\
\text { discussion. }\end{array}$ & $\begin{array}{l}\text { Students } 26 \text { years } \\
\text { and older tend to } \\
\text { have higher marks, } \\
\text { but not statistically } \\
\text { significant }\end{array}$ \\
\hline
\end{tabular}


Table 1: (continued)

\begin{tabular}{|c|c|c|c|c|c|}
\hline Author/Year/Country & $\begin{array}{l}\text { Definition: } \\
\text { mature age/ } \\
\text { academic } \\
\text { success }\end{array}$ & Aim & $\begin{array}{l}\text { Research design/ } \\
\text { sample/data } \\
\text { collection }\end{array}$ & Key findings & Limitations \\
\hline $\begin{array}{l}\text { 16. Nagelsmith, L., } \\
\text { Bryer, J., \& Yan, Z. } \\
\text { /2012/ United } \\
\text { States }\end{array}$ & $\begin{array}{l}\text { No/ Current GPA } \\
\text { drawn from } \\
\text { student } \\
\text { database. }\end{array}$ & $\begin{array}{l}\text { To identify the } \\
\text { relationship among } \\
\text { motivation, volition } \\
\text { and academic success } \\
\text { for adult students }\end{array}$ & $\begin{array}{l}\text { Quantitative: Survey } \\
\text { AVSI \& MSLQ } n=303 \\
\text { Nursing students } \\
\text { Mean age: } 46.75 \\
\text { years Mean for total } \\
\text { GPA: } 3.15\end{array}$ & $\begin{array}{l}\text { Significant } \\
\text { relationships between } \\
\text { motivation, volition } \\
\text { and academic } \\
\text { success. }\end{array}$ & $\begin{array}{l}\text { GPA as the single } \\
\text { measure for } \\
\text { academic success }\end{array}$ \\
\hline $\begin{array}{l}\text { 17. Newton, J. M., Kelly, } \\
\text { C., Kremser, A., } \\
\text { Jolly, B,. \& Billet, } \\
\text { S./2009/ Australia }\end{array}$ & $\begin{array}{l}\text { "Older (i. e. } \\
\text { those aged } 28 \\
\text { years and } \\
\text { over)"/No }\end{array}$ & $\begin{array}{l}\text { To identify what } \\
\text { motivates individuals } \\
\text { to engage in a nursing } \\
\text { career. }\end{array}$ & $\begin{array}{l}\text { Longitudinal multi } \\
\text { method: Interviews, } \\
\text { surveys and field work } \\
\text { observations } \mathrm{n}=26 \\
\text { second and third year } \\
\text { student nurses, Ages } \\
\text { 19- to mid-50s }\end{array}$ & $\begin{array}{l}\text { Older students } \\
\text { reported higher levels } \\
\text { of intrinsic motivation } \\
\text { to learn. }\end{array}$ & $\begin{array}{l}\text { Data gathered from } \\
\text { one healthcare } \\
\text { organization and } \\
\text { university Self- } \\
\text { report study }\end{array}$ \\
\hline $\begin{array}{l}\text { 18. O’Brien, F., Keogh, } \\
\text { B., Neenan, K. } \\
\text { /2009/Ireland. }\end{array}$ & $>23$ years / No & $\begin{array}{l}\text { To explore the } \\
\text { experiences of pre- } \\
\text { registration mature } \\
\text { BSc Nursing students }\end{array}$ & $\begin{array}{l}\text { Qualitative: Focus } \\
\text { Groups } n=28 \text { Mature } \\
\text { age nursing students }\end{array}$ & $\begin{array}{l}\text { Peer support helped } \\
\text { mature age students } \\
\text { to remain motivated } \\
\text { and continue with } \\
\text { programme. }\end{array}$ & $\begin{array}{l}\text { One cohort of } \\
\text { students from one } \\
\text { university }\end{array}$ \\
\hline $\begin{array}{l}\text { 19. Ofori, R./2000/ } \\
\text { United Kingdom }\end{array}$ & $\begin{array}{l}\text { Five } \\
\text { subdivisions/ } \\
\text { Grades }<40 \% \\
\text { and }>40 \%\end{array}$ & $\begin{array}{l}\text { To explore age on the } \\
\text { performance in } \\
\text { nursing modules. }\end{array}$ & $\begin{array}{l}\text { Quantitative: } \\
\text { Assessment results } \\
\text { and questionnaire } n \\
=222 \text { students: } \\
\text { Mature age or non- } \\
\text { traditional (>25.y.o.): } \\
44.6 \%\end{array}$ & $\begin{array}{l}\text { Mature students more } \\
\text { motivated by intrinsic } \\
\text { goals; prior life } \\
\text { experience gives } \\
\text { mature students a } \\
\text { deep approach to } \\
\text { studying. }\end{array}$ & $\begin{array}{l}\text { Exploratory study, } \\
\text { variables not } \\
\text { controlled }\end{array}$ \\
\hline $\begin{array}{l}\text { 20. Ofori, R. \& Charlton, } \\
\text { J.P./2002/United } \\
\text { Kingdom }\end{array}$ & No/No & $\begin{array}{l}\text { To build \& test a } \\
\text { model of } \\
\text { psychological } \\
\text { processes underlying } \\
\text { nursing students' } \\
\text { academic } \\
\text { performance }\end{array}$ & $\begin{array}{l}\text { Quantitative: Co- } \\
\text { relational Design n } \\
=315 \text { pre-registration } \\
\text { nursing students Age } \\
\text { range } 18-50 \text { years old } \\
\text { Mean age of } 26 \text { years } \\
\text { old. }\end{array}$ & $\begin{array}{l}\text { Older students had } \\
\text { stronger internal } \\
\text { control beliefs. } \\
\text { Relationship between } \\
\text { age and academic } \\
\text { performance is } \\
\text { positively correlated } \\
\text { by support seeking. }\end{array}$ & \\
\hline $\begin{array}{l}\text { 21. Pitt, V., Powis, D., } \\
\text { Levett-Jones, T., \& } \\
\text { Hunter, S. } \\
\text { /2014/Australia. }\end{array}$ & No/No & $\begin{array}{l}\text { To provide a } \\
\text { description of } \\
\text { personal qualities of } \\
\text { newly enrolled } \\
\text { Bachelor of Nursing } \\
\text { students }\end{array}$ & $\begin{array}{l}\text { Quantitative: } P Q A \\
\text { assessment } n=138 \\
\text { nursing students } \\
\text { Mean age: } 27 \text { years } \\
\text { old }\end{array}$ & $\begin{array}{l}\text { Older students have a } \\
\text { higher measure of } \\
\text { self-control, } \\
\text { resilience, empathy, } \\
\text { and more } \\
\text { communitarian in } \\
\text { attitude. }\end{array}$ & $\begin{array}{l}\text { Small sample size in } \\
\text { one university }\end{array}$ \\
\hline $\begin{array}{l}\text { 22. Rassool, G. H. and } \\
\text { S. Rawaf/ } \\
\text { 2007/United } \\
\text { Kingdom }\end{array}$ & No/No & $\begin{array}{l}\text { To determine the } \\
\text { predominant learning } \\
\text { style preferences of } \\
\text { UG nursing students. }\end{array}$ & $\begin{array}{l}\text { Quantitative: Survey } \\
\mathrm{n}=110 \text { nursing } \\
\text { Students Mean age: } \\
32.91\end{array}$ & $\begin{array}{l}\text { Mature students have } \\
\text { learned to be adaptive } \\
\text { in their learning and } \\
\text { developed skills to } \\
\text { meet the demanding } \\
\text { requirements of the } \\
\text { course. }\end{array}$ & $\begin{array}{l}\text { Honey and } \\
\text { Mumford's (2000) } \\
\text { learning styles } \\
\text { questionnaire: not } \\
\text { tested for reliability. }\end{array}$ \\
\hline
\end{tabular}


Table 1: (continued)

\begin{tabular}{|c|c|c|c|c|c|}
\hline Author/Year/Country & $\begin{array}{l}\text { Definition: } \\
\text { mature age/ } \\
\text { academic } \\
\text { success }\end{array}$ & Aim & $\begin{array}{l}\text { Research design/ } \\
\text { sample/data } \\
\text { collection }\end{array}$ & Key findings & Limitations \\
\hline $\begin{array}{l}\text { 23. Salamonson, Y. \& } \\
\text { Andrew, S./2006/ } \\
\text { Australia. }\end{array}$ & $\begin{array}{l}\text { No/ Total } \\
\text { assessment } \\
\text { score in subject } \\
\text { matter }\end{array}$ & $\begin{array}{l}\text { To examine the } \\
\text { influence of age, } \\
\text { ethnicity and part- } \\
\text { time employment on } \\
\text { nursing students' } \\
\text { academic } \\
\text { performance }\end{array}$ & $\begin{array}{l}\text { Quantitative: Survey } \\
\mathrm{n}=267 \text { nursing } \\
\text { students Mean age: } \\
25\end{array}$ & $\begin{array}{l}\text { Students who were } \\
\text { older performed } \\
\text { better academically in } \\
\text { pathophysiology and } \\
\text { nursing subjects. }\end{array}$ & $\begin{array}{l}\text { Focused on second } \\
\text { year nursing } \\
\text { students in one } \\
\text { university }\end{array}$ \\
\hline $\begin{array}{l}\text { 24. Steele, R, Lauder, } \\
\text { Caperchione, C. \& } \\
\text { Anastasi, J, } \\
\text { /2005/Australia }\end{array}$ & No/No & $\begin{array}{l}\text { To explore the } \\
\text { problems of mature } \\
\text { students undertaking } \\
\text { an access to nursing } \\
\text { course and to } \\
\text { describe their coping } \\
\text { strategies }\end{array}$ & $\begin{array}{l}\text { Qualitative: In-depth } \\
\text { interviews } n=8 \\
\text { students Age range: } \\
26 \text { years old or over }\end{array}$ & $\begin{array}{l}\text { Students reported } \\
\text { difficulty during their } \\
\text { course; Finance, } \\
\text { Academic, and } \\
\text { balance work/life. } \\
\text { Coping strategies } \\
\text { adopted: Support } \\
\text { Network; Prioritisation } \\
\& \text { organisation; } \\
\text { Positive expectations } \\
\text { and attitudes for the } \\
\text { Future. }\end{array}$ & $\begin{array}{l}\text { Small sample size at } \\
\text { one university }\end{array}$ \\
\hline $\begin{array}{l}\text { 25. Stewart, L. et al. } \\
\text { /2001/ Australia }\end{array}$ & No/No & $\begin{array}{l}\text { To identify knowledge } \\
\text { and use of learning } \\
\text { support services }\end{array}$ & $\begin{array}{l}\text { Mixed Method: } \\
\text { Quantitative: Surveys } \\
(n=150) \text { Qualitative: } \\
\text { interviews }(n=12) \\
\text { mature age students } \\
\text { (<25 years) }\end{array}$ & $\begin{array}{l}\text { Older students tend to } \\
\text { self-refer to learning } \\
\text { support. Younger } \\
\text { students (<25 years) } \\
\text { often lack effective } \\
\text { learning strategies }\end{array}$ & $\begin{array}{l}\text { First year students } \\
\text { only; small sample } \\
\text { size }\end{array}$ \\
\hline $\begin{array}{l}\text { 26. Trotter, E. and G. } \\
\text { Cove/2005/United } \\
\text { Kingdom }\end{array}$ & $>21$ years old/No & $\begin{array}{l}\text { To determine the } \\
\text { issues that impact on } \\
\text { students' early } \\
\text { experience of } \\
\text { university }\end{array}$ & $\begin{array}{l}\text { Mixed Method } \\
\text { Quantitative: Program } \\
\text { documents } \\
\text { Qualitative: Interview } \\
\mathrm{n}=35 \text { first-year } \\
\text { healthcare \& social } \\
\text { students. }\end{array}$ & $\begin{array}{l}\text { Complexity in learning } \\
\text { styles requires } \\
\text { teaching styles that } \\
\text { are applicable for } \\
\text { younger and mature } \\
\text { age students. }\end{array}$ & $\begin{array}{l}\text { Small sample size } \\
\text { limited to one } \\
\text { university }\end{array}$ \\
\hline
\end{tabular}

\section{Age and academic success}

There are several studies that demonstrate a strong relationship between age and academic success. El Ansari (2002) found that age is both positively and significantly correlated with grade achieved $(\mathrm{r}=0.277, p=0.0001)$. In similar results, Salamonson and Andrew (2006) conducted a survey ( $\mathrm{n}=267$ of second year nursing students) to identify the influence of age, gender, ethnicity and part-time employment on nursing students' academic performance. Results indicated that age was positively related with academic performance based upon total assessment score in subject matter.
In contrast, McCarey etal. (2007) observed that mature age students (>26 years old) did not achieve higher marks in coursework and examinations irrespective of entry university qualifications in a study of 154 nursing diploma students at a Scottish University. In another quantitative study conducted by Freitas and Leonard (2011) based upon Maslow's hierarchy of needs, no significant relationship was found between GPA and age (Freitas \& Leonard, 2011).

Nevertheless, eight out of 12 studies that examined the relationship between age and academic performance found that mature age nursing students perform better academically (El Ansari, 2002; Fernandez et al., 2012; 
Madiagn, 2006; McCarey et al., 2007; Nagelsmith et al., 2012; Ofori, 2000; Salamonson \& Andrew, 2006; Ofori \& Charlton, 2002). The factors that positively affect mature age nursing students' academic success can be classified as intrinsic and extrinsic. The following section presents each of these factors.

\section{Intrinsic factors: Life experiences, emotional intelligence, motivation and volition}

A research study composed of educators, state government agents, industry members and student representatives within the State of Victoria in Australia concluded that despite the difficulty in balancing life and study, mature age students were academically successful because of their life experience (Kenny et al., 2011). Selfcontrol and resilience in mature age students allows them to overcome obstacles during the journey of a nursing student (Pitt et al., 2014). Kevern and Webb (2004) conducted group interviews with mature age female nursing students $(n=32)$ with a mean age of 41 years. Despite the struggles mature age nursing students faced in returning to university and maintaining a family life, they reported that life experience gave them the skills they needed to succeed in their studies, in particular, 'persistence' (Kevern \& Webb, 2004; Newton et al., 2009).

Other studies (Fernandez et al., 2012; Jones-Schenk \& Harper, 2014) have found that emotional intelligence (EI) is significantly related to academic achievement. Fernandez et al., (2012) examined the relationship using Motivated Strategies for Learning Questionnaire (MSLQ) between Emotional Intelligence (EI) and academic success among 81 mature age students enrolled in an accelerated nursing programme at an Australian University with a mean age of 29 years. The students scored 148 out of 204 in EI, which was significantly higher than a cohort of traditional high school leavers. Researchers also found a significant correlation between EI scores and critical thinking $(\mathrm{r}=0.41 ; p<0.001)$, help seeking $(\mathrm{r}=0.33 ; p<0.003)$ and peer learning $(\mathrm{r}=0.32$; $p<0.004)$, which indicated that EI is a significant predictor of academic achievement ( $\beta$ 0.25; $\mathrm{p}=0.023)$.

Nagelsmith et al. (2012) measured how volition and motivation correlated with academic success by utilising Academic Volitional Strategies Inventory (AVSI) and Motivated Student Learning Questionnaire (MSLQ). Acceptable measures of validity and reliability have been established for AVSI (McCann \& Turner, 2004). MSLQ motivational scales demonstrated an acceptable level of validity on expectancy component $(\alpha=0.93)$, however; the value-component-extrinsic goal orientation $(\alpha=0.62)$ was less reliable. The study consisted of 303 baccalaureate nursing students who responded to a questionnaire consisting of 20 statements in AVSI and 31 statements in MSLQ describing actions or reasons behind their intent to study. The mean age of participants was 46.8 years and mean GPA was 3.15 out of 4.0. The results indicated that there is a significant relationship between motivation and volition, and academic success.

\section{Extrinsic factor 1: Peer, academic and family support}

The support from peers, academics and family was identified as a foundation to success in mature age nursing students. Peer networks and support help students meet demands, deadlines, and challenges of the courses (Boelen \& Kenny, 2009; Kevern \& Webb, 2004; Steele et al., 2005). Fleming and McKee (2005) observed that an extensive network supported mature age students $(n=130)$ which includes other mature age students (45\%), friends (17.5\%) and family (12.5\%). This finding is supported by a qualitative study (three focus groups with 28 students) conducted by O'Brien et al. (2009) that a positive peer support system assists the students to remain motivated and to continue through the demands of a nursing program.

Academic staff and learning support provide educational support and encouragement building an additional layer to a support system. When mature age students work closely with academic staff who are committed to their jobs, the mature age students demonstrated a deeper understanding of their needs, allowing more opportunities to succeed (Bowden, 2008; Steele et al., 2005). Older students are more likely to seek learning support from campus services (Ofori \& Charlton, 2002; Stewart et al., 2001). Ofori (2000) found that mature age students utilize one-on-one tutorials and build relationships with teachers to further develop study skills. This type of approach develops a student for academic success and for a deeper life-long learning. Ofori and Charlton (2002) noted a direct relationship between support seeking, age, and academic performance among 315 pre-registration nursing students in a university in United Kingdom (UK). They concluded that the ability to seek outside help for academic support reinforced internal control beliefs of the mature age students.

Family support is another factor of academic success in mature age students. Steele et al (2005) explored the problems of mature age nursing students with family/life 
balance. The fine art of balancing family life and academic requirements to complete the nursing program was reinforced by the mature age students' motivation to provide a secure financial future for their families. Kenny et al. (2011) concluded that an informed, supportive family system would provide positive reinforcement to mature age students.

\section{Extrinsic factor 2: Learning style, course content and mode of delivery}

The academic success of mature age students may be explained by preferred learning style, course content, and mode of delivery. Rassool and Rawaf (2007) examined the predominant learning style preferences of undergraduate nursing students $(n=110)$ using the learning styles questionnaire developed by Honey and Mumford (2000). Whilst 'reflector' is the preferred learning style of younger age undergraduate nursing students, mature age students adapt and excel with dual learning styles due to the fact that nurses are both people-orientated (reflector) and scientific (theorist). Rassool and Rawaf (2007) stated that their research may be limited as their original questionnaires were not tested for reliability. Nevertheless, internal consistency in their study was quite high and the study provided nurse academics with the insight that mature age nursing students' preferred learning style is different to that of younger cohorts.

El Ansari (2002) established the importance of course content and mode of delivery, for academic success in higher education among mature age nursing students. A quantitative study design was applied to collect information regarding student satisfaction with healthcare modules in Term 1 of their first academic year at Oxford Brookes University (UK). Older mature students ( $>25$ years old) and mature students (21-25 years old) who had achieved a higher score also had a higher satisfaction with the modules than the younger cohorts ( $<21$ years old). The findings suggest that satisfaction with course content is vital to the success of a mature age student. The older students found that satisfaction was based upon the following components of the modules: wellpresented and organised material, readily available information about module, subject matter relevant to their career and insight into the nursing profession.

Mature age students require an academically challenging environment that respected their life experiences and achievements, and this should be reflected in mode of delivery. Anderson and Thorpe (2008) found that mature age students $(n=754)$ who participated in an inter-professional learning event (IPE) at three universities in England often looked bored as they were already aware of how to do teamwork. In addition, Clarke et al. (2007) found that mature age students (>21 years old) accepted more responsibility for class discussion by encouraging all members to participate. However, at times they may have dominated the group, impeding younger students' participation (Clarke et al., 2007).

\section{Discussion and recommendations}

It is convincing that mature age nursing students are more likely to succeed than a younger age cohort. However, it is not yet conclusive for several reasons. Despite the numerous studies suggesting mature age students succeed academically, there are conflicting definitions of 'mature age' student or the measurement of 'academic success'. The lack of consensus leads to questionable results of some of the recent research literature on mature age nursing students. First of all, the terms 'mature age student' or 'adult learner' in studies examining academic success of mature age nursing students are often defined using different age groups depending on the research study, higher institution, or country. In this review, half of the articles did not provide a definition for mature age student. This inconsistency may possibly affect the statistical comparisons or data analysis of a student population study. Secondly, it is apparent that the definition of 'academic success' varies among researchers and academics. The measure of academic success is traditionally the grade point average (GPA) (Timer \& Clauson, 2011). However, no evidence was found in this review that indicates that the GPA is a valid measurement of academic success despite its use for research purposes. Lastly, the methodological issues noted include that half of the studies were quantitative with small sample sizes, which reduced statistical significance, and the reliability and validity of the tools used in those quantitative studies are either in question or not discussed. In order for future research in this topic to be valid, it is recommended that consensual definitions of 'mature age students' and 'academic success' be developed. Use of measurement tools with high reliability and validity, and larger samples is also warranted.

Studies reviewed in this paper suggest that mature age students are academically successful despite the difficulty in balancing family commitment, work, and study. The key factors associated with academic success in mature age students include a number of extrinsic factors such as additional support from peers, academic staff 
and family members. It is also noted that high achieving mature age students reported a high level of satisfaction with well-designed courses. In addition to extrinsic factors, intrinsic factors derived from students' own life experiences, motivation, and volition also play an important role in their success.

With this in mind, there have been suggestions that age be factored into entry qualifications for a nursing program and there have been attempts to build evidence. For example, the findings from a recent survey $(n=138$, mean age 27) conducted by Pitt etal. (2014) using Personal Quality Assessments (PQA) proposed the new argument that personal qualities are age and gender specific. They found significant correlations with older students having a higher measure of self-control, resilience, empathy, and a more communitarian attitude, which were identified as intrinsic factors for academic success. Personal qualities were unchanged over the three years of their nursing program. This finding is consistent with the significant relationship between a student's ability to control frustration in academic pursuits and obtaining a high GPA (Wilde, 2012).

An argument emerging in relation to mature age nursing students and their academic success is that a student with higher emotional intelligence (EI) exhibits self-awareness and self-reflection that are prominent characteristics of a mature age nursing student. A number of authors (Codier \& Odell, 2014; Fernandez et al., 2012; Jones-Schenk \& Harper, 2014) have suggested that EI is a strong predictor of academic achievement. More interestingly, Benson et al (2012) suggest that EI changes little over the course of a nursing program. This indicates that EI presents a compelling adjunct to current selection criteria for nursing students (Benson et al., 2012). However, the lack of research prevents widespread adoption of this criterion (Jones-Schenk \& Harper, 2014). Future studies should focus on a greater understanding of mature age students' intrinsic motivation and EI, and how these personality traits are reinforced by supportive networks to ensure success in academic pursuit.

The searching for a relationship between 'mature age nursing students and academic success' is problematic due to the dichotomy of extrinsic versus intrinsic. First of all, the extrinsic factors of dedicated academic staff, adult learning styles, and well-designed courses are only one part of the equation to academic success in mature age nursing students. The problem is that these extrinsic factors need to be coupled with the intrinsic determination of a mature age student. For example, the extrinsic factor of course content and mode of delivery needs to be aligned with the teaching component that provides insight into the mature age student's style of learning and motivation (Shelton, 2012). This is well captured in a study by Sogunro (2015) that established eight motivating factors for adult learners in higher education: quality of teaching and curriculum, relevance and pragmatism, interactive classrooms, effective management practices, progressive assessment and timely feedback, self-directedness, learning environment, and effective academic advising practices. A positive peer support system and meaningful relationships with academic staff influences the mature age nursing students' motivation to continue and succeed in a nursing program (Bernard, 2015; Moe et al., 2009; O’Brien et al., 2009). The combination of extrinsic and intrinsic factors of mature age students is relevant for successful academic achievement. Hence, further research is recommended to explore this comprehensive and inclusive relationship to capture the whole picture of mature age students and their academic performance.

It is inevitable that there will be younger age cohorts in a learning setting despite the increasing number of mature age students in nursing programs. Theoretically, mature and younger cohorts in same classroom complement the learning capabilities of each other. An academic environment needs to be developed to simultaneously challenge the older students and support the younger students to engage in the learning process. Nurse educators need to create an environment where senior mature age students could mentor junior students with coping strategies such as resilience as way of passing on their knowledge of university work/life balance (Drury et al., 2008; Taylor \& Reyes, 2012). Further investigation is necessary in order to understand the requirements of successful academic performance of both the mature and younger age cohorts.

\section{Limitations}

The search strategy may not have identified all the relevant literature on this topic since the review was restricted to published and English language studies only. This paper aims to be a comprehensive but not an exclusive review of the current available research on mature age nursing students and academic success.

\section{Conclusions}

It is apparent that there is no clear consensus regarding the definition of mature age students and academic 
success. Life experiences, motivation, and volition are prominent elements of academic success; however, there are necessary external factors to reinforce these elements among mature age students. Given the complexity of both the extrinsic and intrinsic factors, the analysis of factors affecting mature age students' academic success in nursing programs provides nursing academics with promising evidence, emerging ideas and new opportunities to explore and implement in education and further research.

Author contributions: Lisa J Hayden: literature search; Lisa J Hayden, Sarah Y. Jeong: literature analysis and interpretation; Lisa J Hayden, Sarah Y. Jeong, \& Carol A. Norton: preparation of manuscript drafts \& critical revision of manuscript.

\section{Source of funding: None}

Conflict of interest: No conflict of interest has been declared by the authors.

\section{References}

Anderson, E., \& Thorpe, L. (2008). Early interprofessional interactions: Does student age matter. Journal of Interprofessional Care, 22(3), 263-282.

Australian Bureau of Statistics (ABS). (2013). Australian social trends: Hitting the books-characteristics of higher education students; Document no 4102.0. Australian Bureau of Statistics, Canberra.

Bannier, B. J. (2010, August). Understanding our adult, undergraduate learners: Designing course for success. Paper presented at the Annual Distance Teaching and Learning Conference, Madison, WI.

Benson, G., Martin, L., Ploeg, J., \& Wessel, J. (2012). Longitudinal study of emotional intelligence, leadership, and caring in undergraduate nursing students. Journal of Nursing Education, 51(2), 95-101. doi:10.3928/01484834-20120113-01

Bernard, J. S. (2015). Student engagement: A principle-based concept analysis. International Journal of Nursing Scholarship, 12(1), 1548-1923. doi:10.1515/ijnes-2014-0058

Boelen, M., \& Kenny, A. (2009). Supporting enrolled nurse conversion - the impact of a compulsory bridging program. Nurse Education Today, 29(5), 533-537. doi:10.1016/j. nedt.2008.11.015

Bowden, J. (2008). Why do nursing students who consider leaving stay on their courses? Nurse Researcher, 15(3), 45-58.

Clarke, B., Miers, M., Pollard, K., \& Thomas, J. (2007). Complexities of learning together: Students' experience of face-to-face interprofessional groups. Learning in Health \& Social Care, 6(4), 202-212.

Codier, E., \& Odell, E. (2014). Measured emotional intelligence ability and grade point average in nursing students. Nurse Education Today, 34(4), 608-612. doi:10.1016/j. nedt.2013.06.007
Drury, V., Francis, K., \& Chapman, Y. (2008). Mature learners becoming registered nurses: A grounded theory model. Australian Journal of Advanced Nursing, 26(2), 39-45.

El Ansari, W. (2002). Student nurse satisfaction levels with their courses: Part I - effects of demographic variables. Nurse Education Today, 22(2), 159-170.

Fernandez, R., Salamonson, Y., \& Griffiths, R. (2012). Emotional intelligence as a predictor of academic performance in firstyear accelerated graduate entry nursing students. Journal of Clinical Nursing, 21(23/24), 3485-3492. doi:10.1111/j.13652702.2012.04199.x

Fleming, S., Mckee, G., \& Huntley-Moore, S. (2011.). Undergraduate nursing students' learning styles: A longitudinal study. Nurse Education Today, 31(5), 444-449. doi:10.1016/j. nedt.2010.08.005

Freitas, F. A., \& Leonard, L. J. (2011). Maslow's hierarchy of needs and student academic success. Teaching \& Learning in Nursing, 31(5), 444-449. doi:10.1016/j.teln.2010.07.004

Hickey, N., Sumsion, J., \& Harrison, L. (2010). Nursing double degrees: A higher education initiative in times of nursing shortages. Australian Journal of Advanced Nursing, 28(1), 52-59.

Honey, P., \& Mumford, A. (2000). The learning styles questionnaire: 80 item version. Maidenhead, Berkshire: Peter Honey Publications Ltd.

Jones-Schenk, J., \& Harper, M. G. (2014). Emotional intelligence: An admission criterion alternative to cumulative grade point averages for prelicensure students. Nurse Education Today, 34(3), 413-420. doi:10.1016/j.nedt.2013.03.018

Kenny, A., Kidd, T., Nankervis, K., \& Connell, S. (2011). Mature age students access, entry and success in nurse education: An action research study. Contemporary Nurse: $A$ Journal for the Australian Nursing Profession, 38(1/2), 106-118. doi:10.5172/ conu.2011.38.1-2.106

Kenny, A., McLennan, J., Nankervis, K., Kidd, T., Connell, S., \& Buykx, P. (2007). Strengthening mature age students access into undergraduate nurse education; Final report. Resource Document. LaTrobe University, Central Gippsland Institute of TAFE. Retrieved 10 June 2015 from \http://www.health.vic.gov. au/_data/assets/pdf_file/0011/185735/LaTrobe_Mature-Age_ Final Report_07.pdf.

Kevern, J., \& Webb, C. (2004). Mature women's experiences of preregistration nurse education. Journal of Advanced Nursing, 45(3), 297-306. doi:10.1046/j.1365-2648.2003.02890.x

Law, M., Stewart, D., Pollock, N., Letts, L., Bosch, J., \& Westmorland, M. (1998a). Critical review form - Quantitative studies. Retrieved 18 December 2014 from www.srsmcmaster.ca/ Portals/20/pdf/ebp/quanreview_form1.doc.

Law, M., Stewart, D., Pollock, N., Letts, L., Bosch, J., \& Westmorland, M. (1998b.). Guidelines for critical review form - Quantitative studies. Canada: The McMaster University Occupational Therapy Evidence-Based Practice Research Group.

Lee, W., Cholowski, K., \& Williams, A. (2002). Nursing students' and clinical educators' perceptions of characteristics of effective clinical educators in an Australian university school of nursing. Journal of Advanced Nursing, 39(5), 412-420. doi:10.1046/ j.1365-2648.2002.02306.x

Letts, L., Wilkins, S., Law, M., Stewart, D., Bosch, J., \& Westmorland, M. (2007a). Critical review form - Qualitative studies (version 2.0). Retrieved 18 December 2014 from http://www.canchild.ca/en/ canchildresources/resources/qualform.pdf. 
Letts, L., Wilkins, S., Law, M., Stuart, D., Bosch, J., \& Westmorland, M. (2007b). Guidelines for critical review form - Qualitative studies (Version 2.0). Retrieved 18 December 2014 from http://srsmcmaster.ca/wp-content/uploads/2015/05/Guidelines-forCritical-Review-Form-Qualitative-Studies.pdf

Madigan, V. (2006). Predicting prehospital care students' first-year academic performance. Prehospital Emergency Care, 10(1), 81-88.

Malacinski, G. M., \& Winterman, B. (2012). Engaging and motivating undergraduate science students in a writing workshop designed to achieve information literacy and professional level competence. International Journal of Arts \& Sciences, 5(6), 397-414.

McCann, E. J., \& Turner, J. E. (2004). Increasing student learning through volitional control. Teachers College Record, 106(9), 1695-1714.

McCarey, M., Barr, T., \& Rattray, J. (2007). Predictors of academic performance in a cohort of preregistration nursing students. Nurse Education Today, 27(4), 357-364.

Moe, K., Brockopp, D., Walmsley, L., Davis, J., Butler, K., Diebold, C., \& Maiti, S. (2009). A pilot project to evaluate the academic performance, abilities, and satisfaction of second-degree students. Nursing Education Perspectives, 30(4), 226-228. doi:10.1016/S0168-8510(09) 70003.x

Nagelsmith, L., Bryer, J., \& Yan, Z. (2012). Measuring motivation and volition of nursing students in non-traditional learning environments. Journal of Nursing Measurement, 20(2), 90-112.

Newton, J. M., Kelly, C. M., Kremser, A. K., Jolly, B., \& Billett, S. (2009). The motivation to nurse: An exploration of factors amongst undergraduate students, registered nurses and nurse managers. Journal of Nursing Management, 17, 392-400. doi:10.1111/j.1365-2834.2008.00945.x

OECD. (2012). Education at a Glance 2012: OECD indicators. Paris: Author, doi:10.1787/eag-2012-en.

O'Brien, F., Keogh, B., \& Neenan, K. (2009). Mature students' experiences of undergraduate nurse education programmes: The Irish experience. Nurse Education Today, 29(6), 635-640.

Ofori, R. (2000). Age and 'type' of domain specific entry qualifications as predictors of student nurses' performance in biological, social and behavioural sciences in nursing assessments. Nursing Education Today, 20, 298-310.

Ofori, R., \& Charlton, J. P. (2002). A path model of factors influencing the academic performance of nursing students. Journal of Advanced Nursing, 38(5), 507.
Pitt, V., Powis, D., Levett-Jones, T., \& Hunter, S. (2014). Nursing students' personal qualities: A descriptive study. Nurse Education Today, 34(5). doi:10.1016/j.nedt.2014.05.004

Rassool, G., \& Rawaf, S. (2007). Learning style preferences of undergraduate nursing students. Nursing Standard, 21(32), 35-41.

Salamonson, Y., \& Andrew, S. (2006). Academic performance in nursing students: Influence of part-time employment, age and ethnicity. Journal of Advanced Nursing, 55(3), 342-349. doi:10.1111/j.1365 2648.2006.03863

Shelton, E. (2012). A model of nursing student retention. International Journal of Nursing Education Scholarship, 9(1), 1-16. doi:10.1515/1548-923X.2334

Sogunro, O. A. (2015). Motivating factors for adult learners in higher education. International Journal of Higher Education, 4(1), 22-37.

Steele, R., Lauder, W., Caperchione, C., \& Anastasi, J. (2005). An exploratory study of the concerns of mature access to nursing students and the coping strategies used to manage these adverse experiences. Nurse Education Today, 25(7), 573-581.

Stewart, L., Mort, P., \& McVeigh, C. (2001). Barriers and gateways: A study of nursing students' utilisation of learning support resources. Nurse Education Today, 21(3), 209-217.

Taylor, H., \& Reyes, H. (2012). Self-efficacy and resilience in baccalaureate nursing students. International Journal of Nursing Education Scholarship, 9(1), 1-13. doi:10.1515/1548923X.2218

Timer, J. E., \& Clauson, M. I. (2011). The use of selective admissions tools to predict students' success in an advanced standing baccalaureate nursing program. Nurse Education Today, 31(6), 601-606. doi:10.1016/j.nedt.2010.10.015

Trotter, E., \& Cove, G. (2005). Student retention: An exploration of the issues prevalent on a healthcare degree programme with mainly mature students. Learning in Health \& Social Care, 4(1), 29-42.

University of Newcastle. (2014). Program success - Undergraduate success. Data extracted from MIS Portal. Australia: Author.

Watson, J. (2008). Nursing: The philosophy and science of caring. Boulder, CO: University Press of Colorado.

Wilde, J. (2012). The relationship between frustration intolerance and academic achievement in college. International Journal of Higher Education, 1(2), 1-8. 